\title{
ON THE EXISTENCE OF THE FUNCTIONAL MEASURE FOR 2D YANG-MILLS THEORY
}

\author{
ROBERT J. BUDZYŃSKI \\ Institute of Theoretical Physics, Warsaw University \\ Hoża 69, 00-681 Warsaw, Poland \\ E-mail: Robert.Budzynski@fuw.edu.pl \\ WITOLD KONDRACKI \\ Institute of Mathematics, Polish Academy of Sciences \\ Śniadeckich 8, 00-950 Warsaw, Poland \\ E-mail: witekkon@impan.gov.pl
}

\begin{abstract}
We prove the existence of the path-integral measure of two-dimensional YangMills theory, as a probabilistic Radon measure on the "generalized orbit space" of gauge connections modulo gauge transformations, suitably completed following the approach of Ashtekar and Lewandowski.

It has been known for some time that two-dimensional Yang-Mills theory is completely solvable, in the sense that expectation values of a natural class of observables (the Wilson loop functions) with respect to the formal path integral "measure" can be explicitly calculated, i.e. reduced to finite-dimensional integrals. This holds both for the theory on the two-plane, and on topologically nontrivial surfaces. However, a very natural question that seems not to have been addressed in generality is whether the formal path integral measure actually corresponds to some well-defined measure, in the sense of measure theory, and if so, what is the carrier space of such a measure. Actually, for the case of the two-plane a positive answer was provided in [4]; however, the case of compact Riemann surfaces presents significant new features that must be dealt with in a different way. As to the second point, it is generally known that it is not correct to assume that the carrier space of the path integral measure for a quantum field theory is the same as the space of classical smooth field configurations; instead, one must take some completion of this space, incorporating distribution-valued fields in some sense. A concrete realization of this idea for theories of connections (i.e. gauge theories and gravity) was proposed by
\end{abstract}

1991 Mathematics Subject Classification: 81T13, 28C20.

The paper is in final form and no version of it will be published elsewhere. 
Ashtekar and Lewandowski [1], and this is the framework we shall use in the present paper.

The main result of this note is the proof that the path integral measure of twodimensional Yang-Mills theory corresponds to a unique, probabilistic Radon measure on the "generalized orbit space". By Radon measure we mean a measure such that for any open set $U$, its measure $\mu(U)$ is equal to the sup of $\mu(K)$ over all compact subsets $K \subset U$. In addition, we prove that the set of probabilistic Radon measures on the generalized orbit space is itself compact in a suitable topology. This may have some implications for the construction of two-dimensional Yang-Mills theory coupled to matter fields [4].

Let $\mathcal{A}$ denote the space of smooth connections on a principal bundle $(P, \pi, \Sigma, G)$ with total space $P$, structure group $G$ (a compact Lie group), the base manifold being a compact two-dimensional manifold $\Sigma$, and $\pi: P \rightarrow \Sigma$ denoting the bundle projection. The gauge group $\mathcal{G}$ is the group of fiber-preserving automorphisms of the bundle, and acts on $\mathcal{A}$ in a natural way.

Consider the Wilson loop functions

$$
W_{R, \gamma}(A)=\operatorname{Tr}_{R}\left(P e^{\oint_{\gamma} A}\right)
$$

where $A \in \mathcal{A}, \operatorname{Tr}_{R}$ denotes the trace in a representation $R$ of the group $G$, and $P e^{\oint_{\gamma}} A$ is the holonomy of the connection $A$ around a closed loop $\gamma$ in $\Sigma$. Being invariant under the action of $\mathcal{G}$ on $\mathcal{A}$, the Wilson loop functions descend naturally to the quotient space $\mathcal{A} / \mathcal{G}$. Moreover, it turns out that the set of Wilson loop functions for all loops $\gamma$ and all representations $R$ separates points of $\mathcal{A} / \mathcal{G}[3]$. We consider now the algebra generated by all Wilson loop functions. Since the group $G$ is assumed compact, traces of representations are bounded, and it follows that the Wilson loop functions are bounded on $\mathcal{A} / \mathcal{G}$. This allows us to equip the algebra of polynomials in the Wilson loop functions with a norm given the by supremum over $\mathcal{A} / \mathcal{G}$. Completing this algebra with respect to such a norm we obtain a commutative $C^{*}$-algebra, that we denote by $C(\mathcal{A} / \mathcal{G})$.

According to the Gelfand-Naimark theorem [2], any commutative $C^{*}$-algebra (with unit) may be regarded as the algebra of all continuous functions on some compact, Hausdorff topological space. This space may be identified with the set of linear, multiplicative, complex-valued functionals on the given algebra, called the spectrum of the algebra. Applying this theorem to the present case, we can define the space of "generalized" gauge-equivalence classes of connections $\overline{\mathcal{A} / \mathcal{G}}$ to be the spectrum of the algebra $C(\mathcal{A} / \mathcal{G})$. The original space $\mathcal{A} / \mathcal{G}$ is naturally embedded in $\overline{\mathcal{A} / \mathcal{G}}$ as a dense subset. It should be stressed here that the compact space $\overline{\mathcal{A} / \mathcal{G}}$ is, by construction, the largest space onto which the Wilson loop functions extend in a natural way.

Of course, none of the above relies in any way on $\Sigma$ being a two-dimensional manifold. However, when $\Sigma$ is a compact two-dimensional manifold (equipped with a measure), the corresponding Yang-Mills theory admits a particularly simple formulation. Namely, the Migdal-Rusakov formulas [5] provide an explicit description of the functional $\mu$ on $\overline{\mathcal{A} / \mathcal{G}}$ given by the functional integral of two-dimensional Yang-Mills theory. Below we will show that this functional determines a true Radon measure on the space $\overline{\mathcal{A} / \mathcal{G}}$.

Let us consider a (finite) triangulation $I$ of the two-dimensional manifold $\Sigma$. We as- 
sume that the edges of the triangulations are smooth curves in $\Sigma$. To any such triangulation there corresponds a subalgebra of $C(\mathcal{A} / \mathcal{G})$, namely the completion of the subalgebra generated by all Wilson loop functions corresponding to loops built from the edges of the triangulation. This algebra may clearly be identified with the algebra of all continuous functions on the space of "lattice connections" modulo "lattice gauge transformations", $C\left(\mathcal{A}_{I} / \mathcal{G}_{I}\right)$ : a lattice connection is a map from the set of (oriented) edges of the triangulation into the group $G$, and a lattice gauge transformation is an action of the cartesian product of $G$ over the vertices of the triangulation on the lattice connection by

$$
U_{i j} \mapsto g_{i} U_{i j} g_{j}^{-1},
$$

where $U_{i j} \in G$ is the value of the lattice connection corresponding to the edge with endpoints $(i, j)$, and $g_{i} \in G$ describes the lattice gauge transformation at vertex $i$. Obviously, the space of lattice connections modulo lattice gauge transformations is, for any finite triangulation, finite dimensional.

As is known from the Gelfand-Naimark theorem [2], any injective $C^{*}$-algebra homomorphism of commutative $C^{*}$-algebras defines a surjective continuous mapping between the corresponding topological spaces (the spectra of these algebras), operating in the opposite direction. Thus in the present case, the canonical embedding of $C\left(\mathcal{A}_{I} / \mathcal{G}_{I}\right)$ as a subalgebra in $C(\mathcal{A} / \mathcal{G})$ defines a surjective continuous mapping $p_{I}$ from the space $\overline{\mathcal{A} / \mathcal{G}}$ onto $\mathcal{A}_{I} / \mathcal{G}_{I}$, the space of lattice connections modulo lattice gauge transformations described above.

Triangulations of the surface $\Sigma$ form a directed set: we shall say that a triangulation $J$ is finer than $I$ if any closed loop that can be constructed from the edges of $I$ can also be constructed from the edges of $J$. In terms of the corresponding subalgebras of $C(\mathcal{A} / \mathcal{G})$, this means that $C\left(\mathcal{A}_{I} / \mathcal{G}_{I}\right)$ is a subalgebra of $C\left(\mathcal{A}_{J} / \mathcal{G}_{J}\right)$. The corresponding continuous surjection of topological spaces, $p_{J, I}: \mathcal{A}_{J} / \mathcal{G}_{J} \rightarrow \mathcal{A}_{I} / \mathcal{G}_{I}$, satisfies

$$
p_{I}=p_{J} p_{J, I} \text {. }
$$

Thus the topological space $\overline{\mathcal{A} / \mathcal{G}}$ carries the natural structure of a projective system.

Consider a function $f \in C(\mathcal{A} / \mathcal{G})$ that is a product of Wilson loop functions,

$$
f=W_{R_{1}, \gamma_{1}} \cdots W_{R_{n}, \gamma_{n}} .
$$

The Migdal-Rusakov formula for $\mu(f)$ may be described as follows. Take any triangulation $I$ such that all the loops $\gamma_{i}$ may be built from the edges of $I$, and compute

$$
\mu(f)=Z^{-1} \int \chi_{R_{1}}\left(U_{\gamma_{1}}\right) \cdots \chi_{R_{n}}\left(U_{\gamma_{n}}\right)\left(\Pi_{F} K\left(U_{\partial F}, S_{F}\right)\right)\left(\Pi_{(i j)} d U_{(i j)}\right),
$$

where $\chi_{R}\left(U_{\gamma}\right)$ is the character of $G$ in representation $R$ evaluated on the holonomy around the loop $\gamma, F$ numbers the faces of the triangulation, $K\left(U_{\partial F}, S_{F}\right)$ is the heat kernel on $G$ evaluated on the holonomy around the boundary of face $F$ with "time" equal to the area of $F$, and the last factor is the product of Haar measures with respect to the parallel transports along the edges of the triangulation. $Z$ is a normalization factor fixed by the requirement that the integral of the constant function equal to 1 be unity.

The above formula may be interpreted as defining, for any triangulation $I$, a measure 
$\mu_{I}$ on $\mathcal{A}_{I} / \mathcal{G}_{I}$ :

$$
\mu_{I}=Z^{-1}\left(\Pi_{F} K\left(U_{\partial F}, S_{F}\right)\right)\left(\Pi_{(i j)} d U_{(i j)}\right) .
$$

As a consequence of the well known fact that the result obtained by applying the MigdalRusakov formula is independent of the choice of triangulation (among those that fulfill the assumed condition that the curves entering in the Wilson loops making up $f$ can be built from the edges of the triangulation), the family of measures $\mu_{I}$ obeys the condition

$$
p_{I J} * \mu_{J}=\mu_{I}
$$

for all pairs of triangulations $I, J$ such that $J$ is finer than $I$ in the sense defined above. The measures $\mu_{I}$ thus form a projective system of measures, we may therefore apply Prokhorov's theorem: the Prokhorov condition is automatically obeyed, as the space $\overline{\mathcal{A} / \mathcal{G}}$ is compact. Our main result follows:

There exists a unique, Borel and probabilistic Radon measure on $\overline{\mathcal{A} / \mathcal{G}}$ such that for any continuous function $f$, the integral of $f$ with respect to this measure is equal to $\mu(f)$.

One immediate consequence of this result is that for any positive (or non-negative) function $f$ on $\overline{\mathcal{A} / \mathcal{G}}$, the integral of $f$ with respect to this measure (which we will also denote by $\mu(f))$ is necessarily positive (respectively, non-negative).

Now we proceed to show that, in a certain natural topology on $C^{*}(\mathcal{A} / \mathcal{G})$ (the space of continuous linear functionals on $C(\mathcal{A} / \mathcal{G})$ ), the set of probabilistic Radon measures is itself compact. The "physical" implications of this fact concern the coupling of twodimensional gauge fields to matter: assuming that the effect of matter fields may be summarized in a (regulator-dependent) modification of the pure Yang-Mills measure (by e.g. a factor of a discretized fermionic determinant) yielding a sequence of probabilistic Radon measures on $\overline{\mathcal{A} / \mathcal{G}}$, compactness of the space of measures guarantees the existence of convergent sub-sequences - i.e., continuum limits of the coupled Yang-Mills-matter system. Of course, nothing may be said on this level of generality about the uniqueness or physically correct behaviour of these limits.

Consider the mapping from $C(\mathcal{A} / \mathcal{G})$ to $C^{*}(\mathcal{A} / \mathcal{G})$, that assigns to each point the Dirac delta functional with support at that point. With respect to the $*$-weak topology on $C^{*}(\mathcal{A} / \mathcal{G})\left(^{1}\right)$ this mapping is continuous, since for any convergent sequence of points in $\overline{\mathcal{A} / \mathcal{G}}$ the values of a function $f \in C(\mathcal{A} / \mathcal{G})$ at the points of the sequence converge to its value at the limit point. As $\overline{\mathcal{A} / \mathcal{G}}$ is a compact space, so is its image in $C^{*}(\mathcal{A} / \mathcal{G})$. This image forms the set of extremal points of the set of probabilistic measures on $\overline{\mathcal{A} / \mathcal{G}}$. According to the Crane-Millman theorem, the set of probabilistic measures, being a convex set, is the convexification of its subset of extremal points. Since the convexification of a compact set is itself compact, it follows that the set of probabilistic measures on $\overline{\mathcal{A} / \mathcal{G}}$ is compact.

The authors wish to thank Prof. S. Rolewicz and Dr. J. Lewandowski for helpful discussions.

$\left.{ }^{1}\right)$ Defined as the weakest topology such that functions $f \in C(\mathcal{A} / \mathcal{G})$, viewed dually as functionals on $C^{*}(\mathcal{A} / \mathcal{G})$, are continuous. 


\section{References}

[1] A. Ashtekar, J. Lewandowski, Projective techniques and functional integration, J. Math. Phys. 36 (1995), 2170-2191.

[2] J. Dixmier, Les $C^{*}$-algèbres et leurs représentations, Gauthier-Villars, Paris, 1969.

[3] J. Fröhlich, Some results and comments on quantized gauge fields, in: Recent Developments in Gauge Theories, G. 't Hooft et al. (eds.), Plenum, 1980, 53-82.

[4] S. Klimek, W. Kondracki, A construction of two-dimensional quantum chromodynamics, Comm. Math. Phys. 113 (1987), 389-402.

[5] B. E. Rusakov, Loop averages and partition functions in $U(N)$ gauge theory on twodimensional manifolds, Modern Phys. Lett. A 5 (1990), 693-703. 